LAW RENCE LIVERMORE N A T IO N A L LABORATORY

\section{Photon Collider Physics with Real Photon Beams}

J. Gronberg, S. Asztalos

November 4, 2005 
This document was prepared as an account of work sponsored by an agency of the United States Government. Neither the United States Government nor the University of California nor any of their employees, makes any warranty, express or implied, or assumes any legal liability or responsibility for the accuracy, completeness, or usefulness of any information, apparatus, product, or process disclosed, or represents that its use would not infringe privately owned rights. Reference herein to any specific commercial product, process, or service by trade name, trademark, manufacturer, or otherwise, does not necessarily constitute or imply its endorsement, recommendation, or favoring by the United States Government or the University of California. The views and opinions of authors expressed herein do not necessarily state or reflect those of the United States Government or the University of California, and shall not be used for advertising or product endorsement purposes. 


\begin{abstract}
Photon-photon interactions have been an important probe into fundamental particle physics. Until recently, the only way to produce photonphoton collisions was parasitically in the collision of charged particles. Recent advances in short-pulse laser technology have made it possible to consider producing high intensity, tightly focused beams of real photons through Compton scattering. A linear $e^{+} e^{-}$collider could thus be transformed into a photon-photon collider with the addition of high power lasers. In this paper we show that it is possible to make a competitive photon-photon collider experiment using the currently mothballed Stanford Linear Collider. This would produce photon-photon collisions in the $\mathrm{GeV}$ energy range which would allow the discovery and study of exotic heavy mesons with spin states of zero and two.
\end{abstract}




\title{
Photon Collider Physics using Real Photon Beams
}

\author{
J. Gronberg, S. Asztalos \\ Lawrence Livermore National Laboratory, \\ L-050, 7000 East Ave., Livermore CA, 94550, USA*
}

November 3, 2005

\section{Introduction}

The basic idea for producing a photon-photon collider through Compton backscattering was proposed more than 20 years ago [1], and implementations for both warm and superconducting accelerators have been under development $[2,3]$. Several years ago the idea to revive the world's only $e^{+} e^{-}$linear collider, the Stanford Linear Collider (SLC), as a linear collider R\&D testbed was proposed [4]. The addition of hardware in the interaction region to focus a laser pulse onto each incoming beam would allow the creation of photon beams. The photon-photon collisions would be in the energy range which would allow the direct creation of heavy quark bound states, $b \bar{b}$ and $c \bar{c}$, with spin quantum numbers $J^{C P}=0$ and 2. Some of these states have not been observed before. In this paper we determine if sufficient luminosity could be generated to observe and study these states.

\section{The Stanford Linear Collider}

The production of photon beams requires an electron accelerator to drive the Compton process. Storage rings are not suited to this application since the Compton process disrupts the electron beam. Only a linear collider, in which each electron bunch is discarded after the Compton process can form the basis of a photon-photon collider. At present, the only available accelerator is the Stanford Linear Collider (SLC). The SLC achieved a peak luminosity of $3 \times 10^{30}$ $\mathrm{cm}^{-1} \mathrm{~s}^{-1}$ before it was shut down. It has been estimated separately [5] that the accelerator could be restarted and various components upgraded to achieve a

${ }^{*}$ This work was performed under the auspices of the U.S. Department of Energy by the University of California, Lawrence Livermore National Laboratory under Contract No. W7405-Eng-48 
peak luminosity of $2 \times 10^{31} \mathrm{~cm}^{-1} \mathrm{~s}^{-1}$. In this paper we assume beam parameters from the machine upgrades detailed in the LINX proposal [4] with the full SLC repetition rate and bunch charge, as shown in Table 1 . This corresponds to a luminosity of $3 \times 10^{31} \mathrm{~cm}^{-1} \mathrm{~s}^{-1}$.

Table 1: SLC machine parameters used in this study.

\begin{tabular}{|l|c|}
\hline rep rate & $120 \mathrm{~Hz}$ \\
bunch charge & $4.0 \times 10^{10} \mathrm{e}$ \\
beam energy & $30 \mathrm{GeV}$ \\
$\beta_{\mathrm{x}, \mathrm{y}}$ & $(8.0,0.1) \mathrm{mm}$ \\
$\gamma \epsilon_{x, y}$ & $(1.6,0.16) \times 10^{-5} \mathrm{~m}$ \\
$\sigma_{z}$ & 100 microns \\
\hline
\end{tabular}

\section{Photon Beam Generation}

The interaction of the electron beam with a laser pulse is modeled using the CAIN [6] program. The CAIN program models the Compton backscattering process including the effect of high laser intensity and tracks the polarization of all particles. Since the electron bunch and the laser pulse are both moving at the speed of light the best photon production rate occurs when the laser pulse and the electron bunch pass through the center of the laser focus at the same time. Most of the laser pulse is unaffected by the interaction with the electron bunch but a high density of laser photons is required at the focus to improve the rate of Compton scattering. Calculations show that the optimal ratio of Compton scattering to laser pulse energy is achieved when the Rayleigh range of the laser focus and the laser pulse width is the same as the bunch length of the electrons. The parameters of the laser pulse are given in Table 2.

Table 2: Laser and focusing optics parameters.

\begin{tabular}{|l|c|}
\hline wavelength & 1.053 microns \\
flash energy & 2.0 Joules \\
Pulse width & $1.8 \mathrm{ps}$ FWHM \\
Raleigh range $_{(x, y)}$ & $(100,100)$ microns \\
CP-IP distance $^{\mid c} 2 \mathrm{~mm}$ \\
\hline
\end{tabular}

The wavelength and pulse duration are set by the laser technology used in the MERCURY laser [7], which is a high average power laser technology being developed Inertial Confinement Fusion. The Rayleigh range defines the depth of focus of the optics and is matched to the 100 micron electron bunch length 
of the Stanford Linear Collider. The CP-IP distance is the separation of the Compton scattering point from the interaction point.

One advantage of using photons is that it is possible to generate multiple high energy photons from each incoming electron. The photon-photon luminosity is a function of the geometrical $e^{+} e^{-}$luminosity of the $\operatorname{SLC}\left(\mathcal{L}_{\text {geom }}\right)$ and the number of photons per incoming electron $\left(k_{\text {photon }}\right)$ generated during the Compton backscattering, as shown in Equation 1.

$$
\mathcal{L}_{\gamma \gamma} \propto \mathcal{L}_{\text {geom }} \times k_{\text {photon }}^{2}
$$

The value of $k_{\text {photon}}$, and thus the total luminosity, can be made arbitrarily large by increasing the laser flash energy. However, the Compton scattering depletes the electron energy, so each subsequent Compton scatter produces lower energy photons. Therefore, for any particular energy a point of diminishing return is reached.

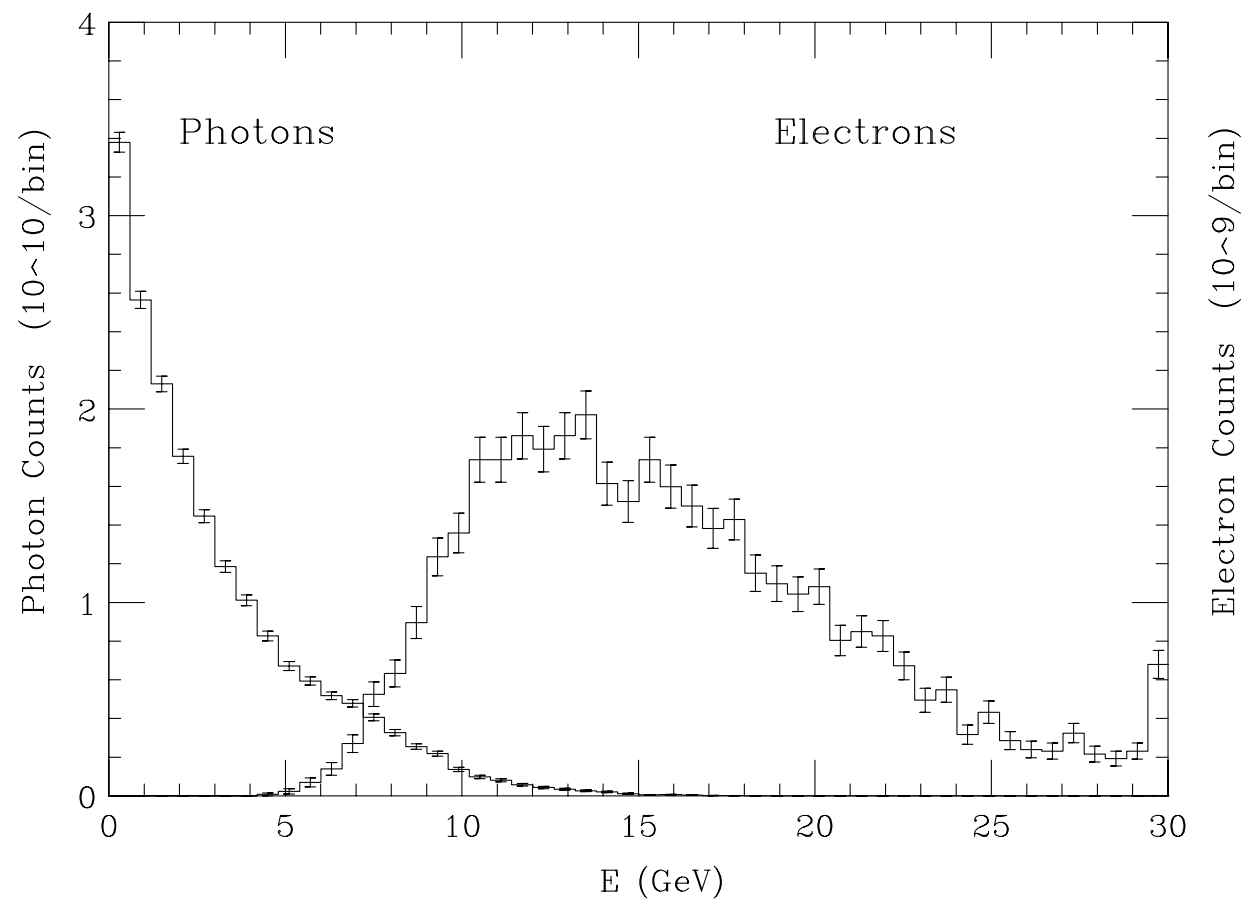

Figure 1: The energy distribution of produced photons and the spent electrons for an input electron bunch of $4 \times 10^{10}$ electrons. (Note the different scale for photons and electrons.)

The photon energy distribution is shown in Figure 1. The laser flash energy of 2.0 Joules allows for multiple Compton scatterings for each electron. 


\subsection{Luminosity Calculation}

The CAIN program tracks the photons and electrons from the conversion point (CP) to the interaction point (IP), taking into account all energy-angle correlations. At the IP the beam-beam effects of the charged particles are simulated and additional "beamstrahlung" photons are generated. The luminosities calculated by CAIN are shown in Figure 2. Since it is assumed that the laser is linearly polarized the photon-photon luminosity divides evenly between spin-0 and spin-2 states.

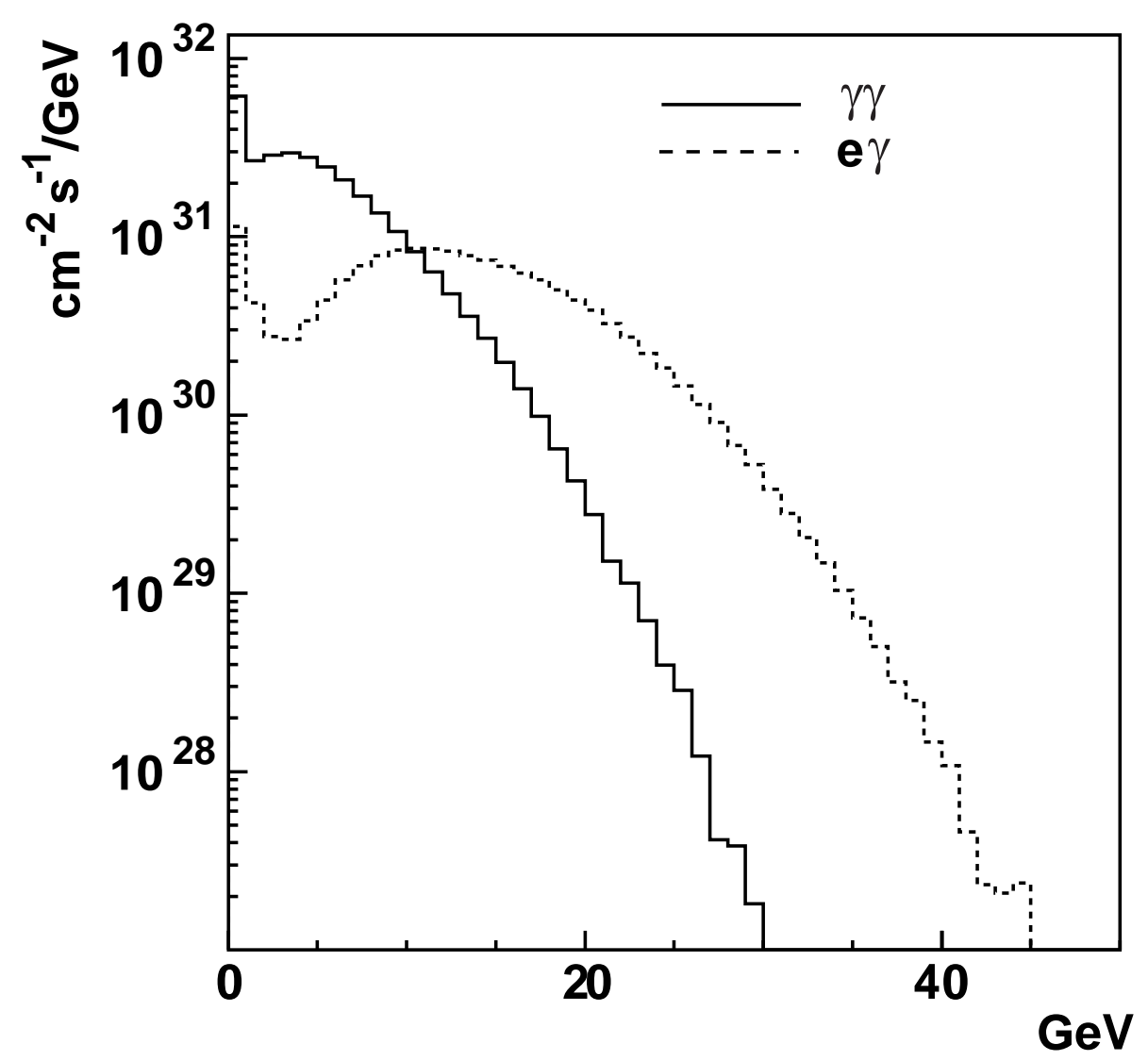

Figure 2: $\gamma \gamma$ luminosity for the beam and laser parameters in Tables 1 and 2 . The total integrated (instantaneous) luminosity is $2.971 \times 10^{32} \mathrm{~cm}^{-2} \mathrm{~s}^{-1}$

$\gamma \gamma$ luminosity is produced when the left- and right-going photons traverse the several millimeters from their respective conversion points to the interaction point. Figure 2 shows the resulting simulated Compton-backscattered $\gamma \gamma$ luminosity at the interaction point from CAIN. For this analysis the luminosity is divided into 50 equally spaced energy bins spanning the range $0<\sqrt{s}<62.4$ 
$\mathrm{GeV}$.

Multiple Compton interactions give rise to significant luminosity above 20 $\mathrm{GeV}$, whereas for lower laser energies (where single interactions dominate) the luminosity is essentially zero for energies above twice the Compton edge. The area under the curve in Fig. 2, or the instantaneous $\gamma \gamma$ luminosity, is $2.971 \times 10^{32} \mathrm{~cm}^{-2} \mathrm{~s}^{-1}$. By way of comparison, the maximum instantaneous $\mathrm{e}^{+} \mathrm{e}^{-}$luminosity at LEP never exceeded $1.0 \times 10^{32} \mathrm{~cm}^{-2} \mathrm{~s}^{-1}$ over its $10+$ years of operation.

\section{Virtual photon luminosity in current experi- ments}

To assess the relative merits of a real photon collider we have considered three colliders whose large $\mathrm{e}^{+} \mathrm{e}^{-}$luminosities would suggest a large virtual $\gamma \gamma$ luminosity: PEP-II [8], KEK-B [9] and LEP [10]. PEP-II and KEK-B are asymmetric $e^{+} e^{-}$colliders running at a center of mass energy of $10.58 \mathrm{GeV}$, while LEP was a symmetric $e^{+} e^{-}$machine that scanned over the energy range $91.2<\sqrt{s} \lesssim$ $206 \mathrm{GeV}$ until decommissioned in late 2000 to make way for the Large Hadron Collider (LHC).

The classical treatment of virtual photons exploits the equivalence between the fields of energetic charged particles and pulses of electromagnetic radiation. In the equivalent photon approximation (EPA) a pulse of electromagnetic radiation is transformed into a frequency spectrum of virtual photons. The interaction of virtual photons from colliding charged particle beams gives rise to virtual photon luminosity. The Weizsacker Williams approximation to the EPA restricts itself to transverse modes of the virtual photon while integrating over the (unobserved) momentum transfer between the electron and the virtual photon to yield [11]

$$
\frac{d N}{d z}=\frac{\alpha}{2 \pi z}\left[\left(1-z+\frac{z^{2}}{2}\right) \log \left(\frac{q_{\max }^{2}}{q_{\min }^{2}}\right)-2 m_{e}^{2}\left(\frac{1}{q_{\min }^{2}}-\frac{1}{q_{\max }^{2}}\right) z^{2}\right]
$$

where $z$ is defined as $\mathrm{E}_{\gamma} / \mathrm{E}_{\text {beam }}, m_{e}$ the electron (or positron) mass, $q_{\min }^{2}$ and $q_{\max }^{2}$ are the minimum and maximum photon energies, respectively, and $d N / d z$ is the number of photons in an energy interval $d z$. Both $q_{\min }^{2}$ and $q_{\max }^{2}$ depend on $z$ in the following manner

$$
\begin{aligned}
q_{\min }^{2} & =\frac{m_{e}^{2} z^{2}}{1-z} \\
q_{\max }^{2} & =(1-z) E_{\text {beam }}^{2} 2\left(1-\cos \theta_{\max }\right)
\end{aligned}
$$

The dependence of $q_{\max }^{2}$ on $\theta_{\max }$ in Eq. 4 merits comment. As $\theta_{\max }$ increases the opportunity for detecting electrons diminishes due to limited detector acceptance, but it is precisely these unobserved electrons that contribute to Eq. 2. The appearance of $\theta_{\max }$ the above expression requires that specific detector configurations be taken into account in the WW analyses. BABAR [12] and 


\begin{tabular}{|c|c|c|c|c|}
\hline & BABAR & BELLE & OPAL & LINX \\
\hline Energy $(\mathrm{GeV})$ & $3.1 / 9.0$ & $3.5 / 8.0$ & $91-206 / 91-206$ & $30 / 30$ \\
\hline$\theta_{\max }(\mathrm{mrad})$ & $523.6 / 295.6$ & $715.6 / 401.4$ & $33 / 33$ & $36 / 36$ \\
\hline
\end{tabular}

Table 3: $e^{+} / e^{-}$beam energies and forward/backward acceptances for the BABAR, BELLE, OPAL and LINX detectors.

\begin{tabular}{|c|c|c|c|c|c|c|}
\hline \multirow{2}{*}{ year } & \multicolumn{2}{|c|}{ BABAR } & \multicolumn{2}{c|}{ BELLE } & \multicolumn{2}{c|}{ OPAL } \\
\cline { 2 - 7 } & peak $^{\perp}$ & int $^{2}$ & peak & int & peak & int \\
\hline \hline 1990 & - & - & - & - & $0.0110^{3}$ & 0.0121 \\
1991 & - & - & - & - & $0.0110^{3}$ & 0.0189 \\
1992 & - & - & - & - & $0.0110^{3}$ & 0.0286 \\
1993 & - & - & - & - & 0.0190 & 0.0400 \\
1994 & - & - & - & - & 0.0231 & 0.0645 \\
1995 & - & - & - & - & 0.0341 & 0.0461 \\
1996 & - & - & - & - & 0.0356 & 0.0247 \\
1997 & - & - & - & - & 0.0570 & 0.0734 \\
1998 & - & - & - & - & 0.0999 & 0.1997 \\
1999 & 1 & 1.62 & $2.05^{4}$ & 0.287 & 0.1069 & 0.2537 \\
2000 & 2 & 23.76 & 2.05 & 10.940 & 0.0675 & 0.2331 \\
2001 & 4.4 & 40.05 & 5.171 & 36.257 & - & - \\
2002 & 5 & 31.32 & 8.256 & 54.181 & - & - \\
2003 & 7.5 & 56.71 & 11.305 & 77.232 & - & - \\
2004 & $7.5^{5}$ & 4.63 & $11.305^{5}$ & 6.827 & - & - \\
\hline \hline Total & - & 158.09 & \multicolumn{1}{|c|}{185.724} & - & 0.9948 \\
\hline
\end{tabular}

Table 4: Instantaneous and integrated $e^{+} e^{-}$luminosities as a function of year. For BABAR and BELLE the 2004 luminosities are through 01/04.

BELLE [13] detectors surround the interaction regions at PEP-II and KEK-B, respectively. We take the OPAL [10] detector as the prototypical LEP detector, while the acceptances of the LINX detector were assumed to be those of the Stanford Linear Detector [14]. Table 3 lists the beam energies and forward and backward acceptances seen by the four detectors under consideration. The forward and backward acceptances of BABAR and BELLE are unequal due to the asymmetric beam energies.

In order to compare real and virtual $\gamma \gamma$ luminosities yearly $\mathrm{e}^{+} \mathrm{e}^{-}$luminosity records for BABAR, BELLE and OPAL were compiled. Table 4 summarizes the peak and integrated $e^{+} e^{-}$luminosities seen by each detector since initiation of their respective operations.

\footnotetext{
${ }^{1}$ In units of $n b^{-1} \mathrm{~s}^{-1}$

${ }^{2}$ In units of $\mathrm{fb}^{-1}$

${ }^{3}$ LEP peak luminosities assumed for 1990-1992.

${ }^{4}$ BELLE peak luminosity not available for 1999.

${ }^{5}$ BELLE and BABAR peak luminosities for 2004 taken from 2003.
} 


\section{Simulation of heavy meson production and decay}

To simulate Weizsacker Williams luminosity the physics event generator PANDORA 2.1 [15] was used. Since Eq. 2 increases without limit as $\mathrm{z} \rightarrow 0$, it was necessary to introduce a low-energy cutoff to regularize the result. An energy cutoff of $1 \mathrm{MeV}$ was employed in all subsequent analyses. The choice of cutoff obviously affects the integrated virtual luminosity, but has no discernible affect on the virtual photon spectra above the energy range of interest $(\sim 3 \mathrm{GeV})$, nor on the luminosity integrated cross sections discussed below. Virtual $\gamma \gamma$ luminosity was calculated for the PEP-II, KEK-B and LEP beams using Pandora in two steps. First, a random number corresponding to the energy of a virtual in one beam was generated. Associated with this photon energy is a specific value of $d N / d z$ from the Weizsacker Williams approximation. This same procedure was repeated for the opposing beam. The product of the $d N / d z$ from each opposing beam gives the relative virtual $\gamma \gamma$ luminosity per unit $e^{+} e^{-}$luminosity, which for PEP-II was determined to be 0.19. Instantaneous virtual $\gamma \gamma$ luminosities were derived by multiplying the relative virtual $\gamma \gamma$ luminosities by the instantaneous $e^{+} e^{-}$luminosities from Table 4 . Although the table contains yearly peak luminosities (when available), for our purpose the peak luminosity with the greatest magnitude was used. A comparison with the LINX instantaneous $\gamma \gamma$ luminosity is shown in the lower graph in Fig. 3. In both graphs in this figure and in the analyses described below the BELLE and BABAR luminosities have been summed together, while the LEP luminosity is that seen by the OPAL detector, but multiplied by four to account for the four LEP detectors. The characteristic rise inherent in the Weizsacker Williams description of virtual photons is seen in the BELLE+BABAR and LEP plots, whereas the rise in the LINX luminosity at low energies stems instead from bremsstrahlung and multiple scattering. Using the relative $\gamma \gamma$ luminosities above and the peak luminosity data from Table 4, one arrives at peak instantaneous luminosities of $3.479 \times 10^{33} \mathrm{~cm}^{-2} \mathrm{~s}^{-1}$ and $3.121 \times 10^{32} \mathrm{~cm}^{-2} \mathrm{~s}^{-1}$ for BABAR+BELLE and LEP, respectively. By comparison, the LINX instantaneous luminosity is $2.971 \times 10^{32} \mathrm{~cm}^{-2} \mathrm{~s}^{-1}$.

A similar approach was used in producing the integrated luminosities in the top graph in Fig. 3, but here the relative virtual $\gamma \gamma$ luminosities are multiplied by the yearly integrated luminosities and summed over relevant years to form a total integrated luminosity. For instance, in the case of LEP there are 11 contributions to the sum (years 1990 to 2000) - each year with its own relative and integrated luminosity to account for the changing beam energy with time. A summary of the results for the normalized, instantaneous and integrated luminosities is contained in Table 5. Taken together, Table 5 and the top graph in Fig. 3 is one of the our main findings: although the integrated virtual $\gamma \gamma$ luminosity from BELLE+BABAR greatly exceeds the integrated real $\gamma \gamma$ luminosity expected from LINX, due to the sharply decreasing nature of the Weizsacker-Williams spectrum this dominance disappears beyond $3.0 \mathrm{GeV}$. As will be seen in the next section, since much of the physics of interest lies above this energy, real $\gamma \gamma$ luminosity offers a very viable approach to heavy meson 


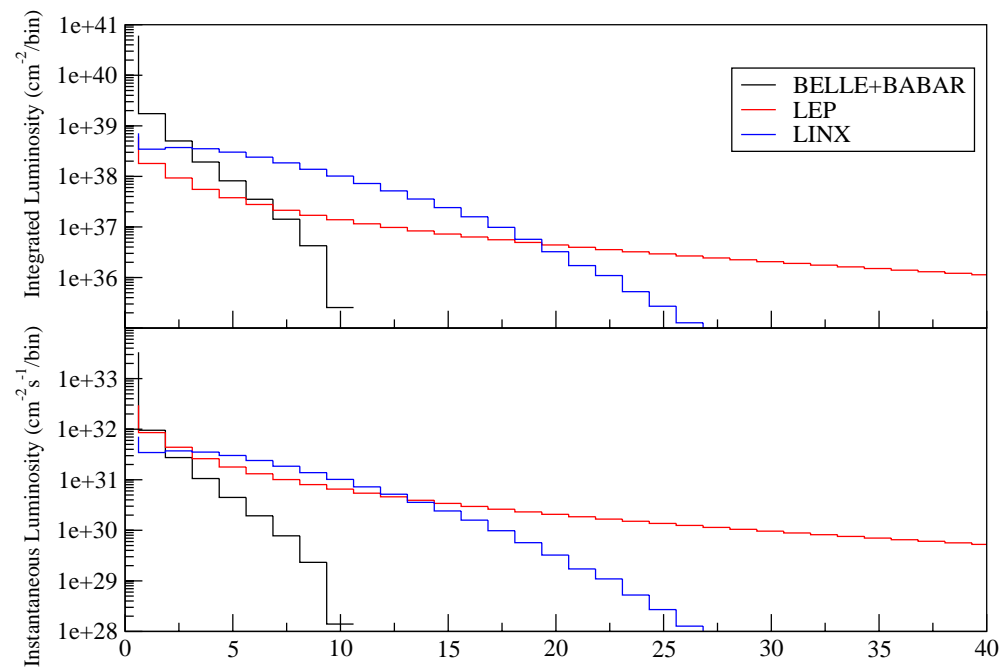

Figure 3: The instantaneous (lower) and integrated (upper) virtual WeizsackerWilliams luminosities seen by BELLE+BABAR and LEP compared to the real luminosity seen at LINX. All luminosities are in $1.3 \mathrm{GeV}$ bins.

\begin{tabular}{|l|c|c|c|}
\hline & BABAR+BELLE & LEP & LINX \\
\hline norm & 0.19 & $0.52-0.70$ & 1.0 \\
\hline inst $\left(10^{32} \mathrm{~cm}^{-2} \mathrm{~s}^{-1}\right)$ & 34.79 & 3.121 & 2.971 \\
\hline intr $\left(10^{39} \mathrm{~cm}^{-2} \mathrm{~s}\right)$ & 63.86 & 2.60 & 2.971 \\
\hline
\end{tabular}

Table 5: Normalized, instantaneous and integrated luminosities from BABAR+BELLE, LEP and LINX. One Snowmass year of operation $\left(10^{7} \mathrm{~s}\right)$ was assumed for the LINX integrated luminosity.

spectroscopy.

\section{Physics Opportunities}

One goal of heavy quark spectroscopy is to test Quantum Chromodynamics predictions of the masses, widths and quantum numbers of these mesons by comparing them with their experimental values. Production of pseudoscalar mesons is a particularly attractive physics goal for a low-energy $\gamma \gamma$ collider since the mesons quantum numbers $\mathrm{J}^{C P}=0^{+-}$preclude them from being produced directly at an $e^{+} e^{-}$collider. Although the lowest radial excitations of the charmonium system has been thoroughly mapped out (with the exception of the singlet $h(1 \mathrm{p})$ state), spectroscopy of the second radial excitation has numerous holes. Even where there have been recent measurements, as in the case 


\begin{tabular}{|c||c|c|c|}
\hline meson & mass & $\Gamma_{\text {tot }}$ & $\Gamma_{\gamma \gamma}$ \\
\hline \hline$\eta_{c}(1 \mathrm{~S})$ & 2.979 & 0.0161 & $7.4 \times 10^{-6}$ \\
\hline$\eta_{c}(2 \mathrm{~S})$ & 3.654 & 0.0161 & $7.4 \times 10^{-6}$ \\
\hline$\chi_{c 0}$ & 3.415 & 0.0107 & $2.6 \times 10^{-6}$ \\
\hline$\eta_{b}(1 \mathrm{~S})$ & 9.3 & 0.014 & $5.3 \times 10^{-7}$ \\
\hline
\end{tabular}

Table 6: Masses and widths of the mesons in GeV.

of the pseudoscalar $\eta_{c}(2 \mathrm{~S})$, disagreement persists as to the excitation (mass). Conflict between experiment and theory on the order of $50 \mathrm{MeV}$ in mass of the $\eta_{c}(2 \mathrm{~S})$ is the source of some consternation in the theoretical community [16]. Enhanced statistics would further allow for the determination of unknown branching ratios. The situation is more severe in the bottom system: none of the pseudoscalar bottom mesons have been definitively observed [17]. Bottom pseudoscalar mesons apparently are more difficult to produce at $e^{+} e^{-}$colliders: their heavier heavier masses imply a more non-relativistic wave function. This, in turn, enhances the selection rule which in the non-relativistic limit forbids $E(1)$ transitions between the $\Upsilon(\mathrm{nS}) \rightarrow \eta_{b}(\mathrm{nS})$.

Using the integrated luminosities described in the previous section we have studied the production of pseudoscalar and other suitable heavy charm and bottom mesons at a low-energy $\gamma \gamma$ testbed and have compared these results to what can be achieved through virtual $\gamma \gamma$ luminosity. Meson simulations were again carried out using the PANDORA event generator. Meson production in PANDORA is modeled using the Breit-Wigner approach; necessary modifications were made to include the widths and masses of the various mesons that are summarized in Table 6. To model meson production at LINX CAIN 2.1 files were imported into PANDORA. These CAIN luminosity files have 10000 entries representing the partitioning of the energy range $0<\sqrt{s}<62.4 \mathrm{GeV}$ into 50 equally spaced energy bins of $1.248 \mathrm{GeV}$ each for $\mathrm{E}_{\gamma 1}$ and $\mathrm{E}_{\gamma 2}$. There are four sets of 2500 entries corresponding to the four helicity combinations possible when two photons interact. Each of the 10000 entries contains $E_{\gamma 1}$ and $E_{\gamma 2}$, luminosity and overall helicity. PANDORA normalizes the luminosity so that the sum over all 10000 entries is 1.0. Meson production at BABAR+BELLE and LEP was modeled using the luminosity class described in Section 4.

PANDORA can be made to output luminosity integrated cross sections for the process of interest, defined as

$$
\bar{\sigma} \equiv \int \frac{\partial L}{\partial E} \sigma d E
$$

In practice, the integral is replaced by a finite sum sampled from a uniformly distributed $\mathrm{E}_{\gamma 1}$ and $\mathrm{E}_{\gamma 2}$ plane. The utility of the luminosity integrated cross section is that when multiplied by the total integrated luminosity the result is the number of events for the process under consideration. Table 7 lists the luminosity integrated cross sections and total number of events produced for each meson in the previous table. Note that unlike the conventional definition of cross section, the luminosity integrated cross section depends on details of the 
environment in which the meson is produced: it is lowest for BABAR+BELLE, for which the integrated luminosity is greatest. From this same table it is seen that number of $\eta_{c}(1 \mathrm{~S})$ events produced at BABAR+BELLE and LINX are approximately equal. Figure 3 provides a consistency check on this result. In the upper plot in that figure the integrated luminosities of BABAR+BELLE and that of LINX are seen to be approximately equal at an energy corresponding to the mass of the $\eta_{c}(1 \mathrm{~S})$. Beyond this crossover point, a greater number of heavier mesons are produced at the LINX facility than at BELLE+BABAR or LEP. For the purposes of bottom spectroscopy the LINX scenario is clearly superior, with the potential for producing an order of magnitude or more $\eta_{b}(1 \mathrm{~S})$ mesons than either BELLE+BABAR or LEP.

In order to simulate experimental reconstruction of these mesons at a $\gamma \gamma$ testbed it is necessary to specify the decay mode, as well as potential backgrounds. Decay of heavy mesons into p $\bar{p}$ pairs was chosen due to the simplicity of the event reconstruction, the relatively large branching ratios and knowledge about the competing background process. The dominant background $\gamma \gamma \rightarrow \mathrm{p} \overline{\mathrm{p}}$ arises when both photons are resolved into hadrons. This process has been well-studied for insight into the photon structure function and resolution of the ongoing debate concerning diquark versus independent quark models of the hadron. Theoretical [18] and experimental [19] efforts have gone into determining the cross section for this process. In particular, the latest BELLE results describe a differential cross section (in $\mathrm{nb} / \mathrm{sr}$ ) for this process of the form

$$
\frac{d \sigma}{d E d(\cos \theta)}=1.15 \times 10^{6} \sigma^{15}\left(\frac{1+\cos ^{2} \theta}{1-\cos ^{2} \theta}\right)
$$

for $|\cos (\theta)| \leq 0.6$ and $\sqrt{s} \geq 2.75 \mathrm{GeV}$. This result is consistent with the diquark model, in contrast to the independent quark model predicts an isotropic angular distribution. The normalization factor that appears in Eq. 6 was derived directly from BELLE data. A separate Pandora class was written to generate the $\gamma \gamma \rightarrow \mathrm{p} \overline{\mathrm{p}}$ background; when convolved with the LINX luminosity described above a luminosity integrated cross section subject to the above kinematic limits of $0.0058 \mathrm{nb}$ is obtained, which corresponds to 17337 events over a Snowmass year of operation. Similarly, to compute the number reconstructed meson events it was necessary to multiply the raw number of meson events from the last row of Table 7 by the branching ratio for decay into $\mathrm{p} \overline{\mathrm{p}}$ pairs. Table 8 summarizes the number of events expected from this procedure. The Phythia interface to Pandora allows events to be written in StdHeP format [20], which in turn

\begin{tabular}{|c|c|c|c|c|c|c|}
\hline \multirow{2}{*}{ meson } & \multicolumn{2}{|c|}{ BABAR+BELLE } & \multicolumn{2}{|c|}{ LEP } & \multicolumn{2}{c|}{ LINX } \\
\cline { 2 - 7 } & $\bar{\sigma}$ & events & $\bar{\sigma}$ & events & $\bar{\sigma}$ & events \\
\hline$\eta_{c}(1 \mathrm{~S})$ & $3.19 \mathrm{e}+4$ & $1.08 \mathrm{e}+7$ & $1.72 \mathrm{e}+6$ & $1.98 \mathrm{e}+6$ & $3.34 \mathrm{e}+6$ & $9.91 \mathrm{e}+6$ \\
\hline$\eta_{c}(2 \mathrm{~S})$ & $1.25 \mathrm{e}+4$ & $4.25 \mathrm{e}+6$ & $8.43 \mathrm{e}+5$ & $9.74 \mathrm{e}+5$ & $1.97 \mathrm{e}+6$ & $5.84 \mathrm{e}+6$ \\
\hline$\chi_{c 0}$ & $5.23 \mathrm{e}+3$ & $1.78 \mathrm{e}+6$ & $3.32 \mathrm{e}+5$ & $3.83 \mathrm{e}+5$ & $7.66 \mathrm{e}+5$ & $2.28 \mathrm{e}+6$ \\
\hline$\eta_{b}(1 \mathrm{~S})$ & $3.05 \mathrm{e}-1$ & $1.09 \mathrm{e}+2$ & $3.25 \mathrm{e}+2$ & $3.89 \mathrm{e}+2$ & $1.07 \mathrm{e}+3$ & $3.17 \mathrm{e}+3$ \\
\hline
\end{tabular}

Table 7: The luminosity integrated cross sections and events produced for the three machine scenarios considered. 


\begin{tabular}{|c||c|c|c|}
\hline meson & BR $(\mathrm{p} \overline{\mathrm{p}})$ & $\mathrm{p} \overline{\mathrm{p}}$ & Reconstructed $\mathrm{p} \overline{\mathrm{p}}$ events \\
\hline \hline$\eta_{c}(1 \mathrm{~S})$ & 0.0012 & $1.19 \mathrm{e}+4$ & $7.09 \mathrm{e}+3$ \\
\hline$\eta_{c}(2 \mathrm{~S})$ & 0.00049 & $2.80 \mathrm{e}+3$ & $1.69 \mathrm{e}+3$ \\
\hline$\chi_{c 0}$ & 0.00024 & $5.47 \mathrm{e}+2$ & $3.32 \mathrm{e}+2$ \\
\hline$\eta_{b}(1 \mathrm{~S})$ & 0.0012 & $4.00 \mathrm{e}+0$ & $3.00 \mathrm{e}+0$ \\
\hline \hline$\gamma \gamma \rightarrow \mathrm{p} \overline{\mathrm{p}}$ & - & $1.73 \mathrm{e}+4$ & $1.73 \mathrm{e}+4$ \\
\hline \hline Total & - & $3.26 \mathrm{e}+4$ & $2.64 \mathrm{e}+4$ \\
\hline
\end{tabular}

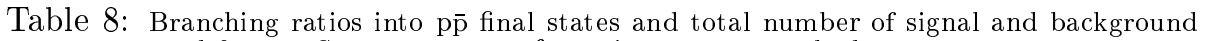
events expected for one Snowmass year of running at a $\gamma \gamma$ testbed.

are read in by the LCDROOT [21] - a detector simulation and analysis framework based on ROOT [22]. In this analysis the p $\bar{p}$ final states and background were propagated through a small version of a hypothetical NLC detector whose electromagnetic and hadronic calorimeters reside in $5 \mathrm{~T}$ magnetic field. Details of the detector [23] are not crucial to the results. Passage of p $\bar{p}$ through the detector simulation results in momenta smearing. In the hadronic calorimeter the momenta is inferred. For this analysis only inclusive decays to $\mathrm{p} \overline{\mathrm{p}}$ are considered, thus all particles reaching the hadronic calorimeters were assumed to have the mass of a proton. With this information and the total energy measured in the electromagnetic calorimeter the four vector is constructed and the invariant mass is calculated. Figure 4 is a reconstruction of the invariant mass of

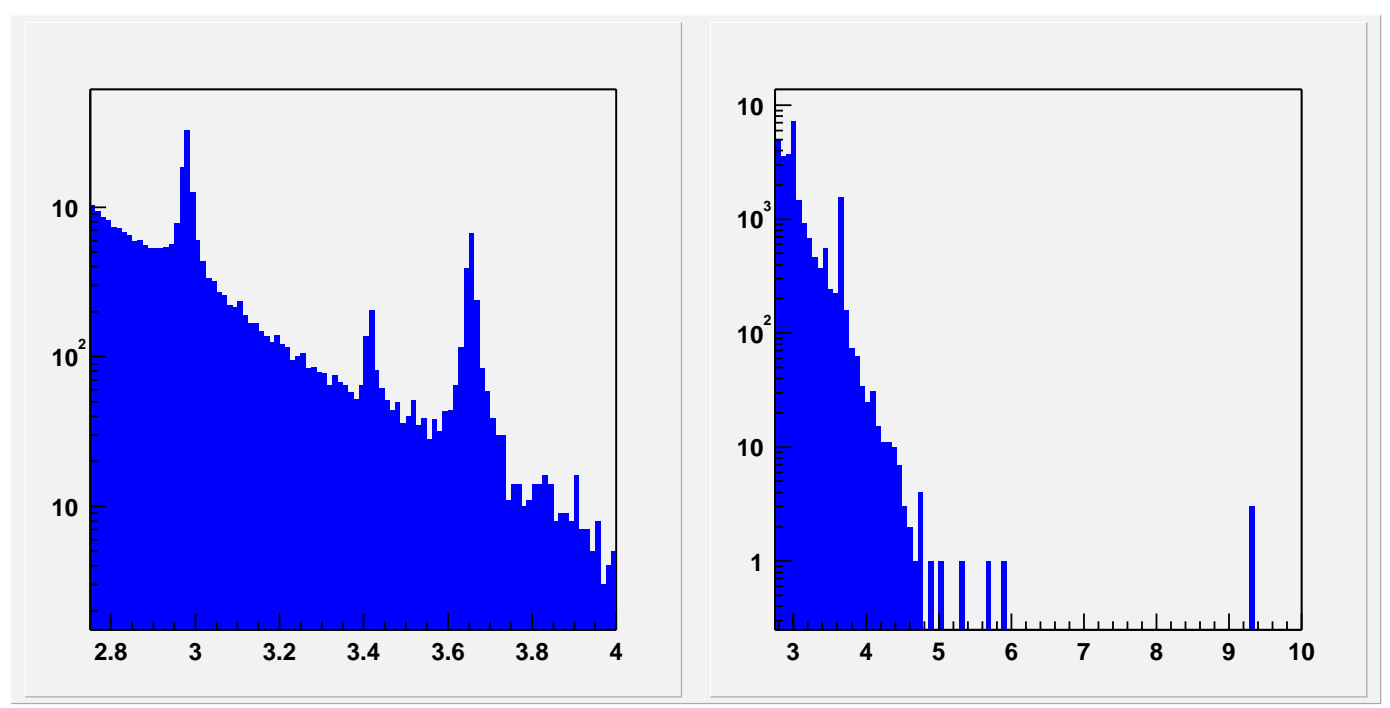

Figure 4: Reconstructed meson and background events expected from a Snowmass year of operation at LINX. Lower and upper plots are identical except for the scaling of the axes. Cuts of $|\cos (\theta)| \leq 0.6$ and $\sqrt{s} \geq 2.75 \mathrm{GeV}$ were applied to all data. 
events leaving a track in the hadronic calorimeter subject to the restriction that $|\cos (\theta)| \leq 0.6$ and $\sqrt{s} \geq 2.75 \mathrm{GeV}$. The total number of reconstructed events $(26,494)$ found in the last column of 8 is significantly fewer than the 32,527 StdHeP events input into LCDROOT. This is an artifact stemming from the fact that kinematic cuts were not imposed on the meson events within PANDORA but rather inside LCDROOT. For the isotropically decaying mesons the fraction lost is consistent with the restricted phase space imposed by the angular cuts. The meson events clearly stand out above the $\mathrm{p} \overline{\mathrm{p}}$ background.

\section{Hardware}

The SLC and its detector require some modifications to be converted to a photon-photon collider. These involve providing a laser, focusing its laser pulses within the confined space of the detector and handling the highly disrupted spent electron beams.

\subsection{Interaction Region Optics}

Space is available with replacement of the SLD beampipe for focusing mirror to be able to focus the laser pulses within $2 \mathrm{~mm}$ of the interaction point. This would necessitate co-locating the optics in the accelerator vaccum.

\subsection{Laser}

Making a conservative estimate of $20 \%$ power loss between the laser and the focal point and including a factor of 2 for two conversion points we can calculate the average laser power required to be:

$$
\frac{2 \mathrm{~J} \times 2 \mathrm{CPs} \times 120 \mathrm{~Hz}}{0.8 \text { efficiency }}=600 \mathrm{~W}
$$

The MERCURY laser [7] is designed to have an average power of $1 \mathrm{~kW}$ and its prototype has already been operated with pulses of $55 \mathrm{~J} \times 10 \mathrm{~Hz}=550 \mathrm{~W}$. Therefore, there exists a laser that has already demonstrated the required average power.

\subsection{SLC modifications}

Aside from the upgrades to increase the SLC's geometric luminosity, there are several modifications that must be made in order to handle the spent beams. The incoming electron beam is essentially monochromatic at $30 \mathrm{GeV}$. Each electron loses energy during the Compton scattering leading to a final distribution of energies as shown in Figure 1. About half of the incoming beam energy is transfered to the Compton photons. The large value of the electron energy spread makes traditional steering optics difficult and, of course, the Compton

photons cannot be steered at all. One solution for the spent beams would be to 
include a crossing angle such that the spent electrons could travel in a straight line to the beam dump. This would require a new beam dump and extraction line tunnel.

\section{Conclusion}

A photon-photon collider experiment based on the SLC would be able to do precision studies of $c \bar{c}$ mesons and would be the most likely way to discover and study $b \bar{b}$ mesons of spin quantum numbers zero and two. The power requirements for the laser are within the range of currently existing lasers. This concept was presented at the SLAC 20-year scenario study and was accepted as technically viable and capable of interesting physics but was considered too expensive to pursue given the current state of the HEP budget.

\section{References}

[1] I. Ginzburg, G. Kotkin, V.Serbo and V. Telnov, Pizma ZhETF 34 (1981) 514; JETP Lett. 34 (1982) 491 (Preprint INF 81-50, Novosibirsk (1981) in English).

[2] The NLC Design Group, "Zeroth Order Design Report for the Next Linear Collider", LBNL-5424, SLAC-474, UCRL-ID-124161, UC-414 (1996).

[3] The TESLA Collaboration, "TESLA Technical Design Report", DESY2001-011, ECFA-2001-209, DESY-TESLA-2001-23, DESY-TESLA-FEL2001-05, Mar. 2001.

[4] http://www-project.slac.stanford.edu/lc/linx

[5] T. Raubenhiemer, "High Energy Accelerators for the SLAC site", Talk presented during the SLAC Scenario Study. http://wwwproject.slac.stanford.edu/lc/local/talks/ shops\%20tor3.pdf

[6] K. Yokoya, "A Computer Simulation Code for the Beam-Beam Interaction in Linear Colliders", KEK report 85-9, Oct. 1985.

[7] L. Hackel, et al., UCRL-TB-136126-01-02.

[8] http://www.slac.stanford.edu/accel/pepii/home.html

[9] http://belle.kek.jp/

[10] http://opal.web.cern.ch/Opal/

[11] K. F. von Weizsacker, Z. Physik 88, 612 (1934), E. J. Williams, Phys. Rev. 45, (1934) 729.

[12] http://www.slac.stanford.edu/BFROOT/ 
[13] http://belle.kek.jp/

[14] http://www-sld.slac.stanford.edu/sldwww/sld.html

[15] http://www-sldnt.slac.stanford.edu/nld/new/Docs/Generators/PANDORA.htm

[16] DELPHI Collaboration, J. Abdallah, et al, Eur. Phys.J. C31 (2003) 481

[17] ALEPH Collaboration, Phys.Lett. B530, (2002) 56

[18] V. M. Budnev et al., Phys. Rep. 15, (1975) 181

[19] Ch.-Ch. Kuo et al., Nucl. Phys.B (Proc. Suppl.) 126, (2004) 313

[20] http://www-cpd.fnal.gov/psm/stdhep/

[21] http://www-sldnt.slac.stanford.edu/nld/New/Docs/LCD_Root/root.htm

[22] http://root.cern.ch/

[23] http://wwwal.kuicr.kyoto-u.ac.jp/www/accelerator/sspm/Iwasaki.pdf 